\title{
EPIDEMIOLOGICAL AND VIROLOGICAL ASSESSMENT OF INFLUENZA ACTIVITY IN EUROPE, DURING THE 2006-2007 WINTER
}

\author{
J MS Arkema (a.arkema@nivel.nl) ${ }^{1}$, A Meijer ${ }^{1}$, T. J. Meerhoff ${ }^{1}$, J Van Der Velden ${ }^{1,2}$, W J Paget ${ }^{1}$, European Influenza \\ Surveillance Scheme (EISS) ${ }^{3}$ \\ 1. European Influenza Surveillance Scheme Co-ordination Centre, Netherlands Institute for Health Services Research (NIVEL), \\ Utrecht, the Netherlands \\ 2. Radboud University Nijmegen Medical Center, Nijmegen, the Netherlands \\ 3. Members of EISS (2006-2007 winter), see article supplement
}

Influenza surveillance in Europe is based on influenza surveillance networks that cooperate and share information through the European Influenza Surveillance Scheme (EISS). EISS collected clinical and virological data on influenza in 33 countries during the 2006-2007 winter. Influenza activity started around 1 January and first occurred in Greece, Scotland and Spain. It then moved gradually across Europe from south to north and lasted until the end of March. In 29 out of 33 countries, the consultation rates for influenza-like-illness or acute respiratory infections in the winter of 2006-2007 were similar or somewhat higher than in the 2005-2006 winter. The highest consultation rates for influenzalike-illness were generally observed among children aged 0-4 years and 5-14 years. The predominant virus strain was influenza $A$ ( $97 \%$ of total detections) of the H3 subtype (93\% of H-subtyped A viruses; $7 \%$ were $A(H 1)$ ). The influenza $A(H 3)$ and $A(H 1)$ viruses were similar to the vaccine reference strains for the 2006-2007 season, A/Wisconsin/67/2005 (H3N2) and A/New Caledonia/20/99 $(\mathrm{H} 1 \mathrm{~N} 1)$ respectively. The majority of the influenza $\mathrm{B}$ viruses were similar to the reference strain B/Malaysia/2506/2004, included in the 2006-2007 vaccine.

In conclusion, the 2006-2007 influenza season in Europe was characterised by moderate clinical activity, a south to north spread pattern across Europe, and a dominance of influenza A(H3). Overall there was a good match between the vaccine virus strains and the reported virus strains.

\section{Introduction}

Influenza is an acute self-limiting viral disease of the upper respiratory tract. Influenza has a considerable public health impact in Europe each winter because of its ability to spread rapidly through populations by coughs and sneezes from infected people [1].

During seasonal influenza epidemics $5-15 \%$ of the population are affected with upper respiratory tract infections [2]. Seasonal epidemics are associated with substantial demands on healthcare resources and considerable costs due to increases in general practice consultation rates, clinical complications, hospitalisations, drug treatment and absence from work $[3,4]$. Although difficult to assess, it is estimated that worldwide between 250,000 and 500,000 people die from severe illness as a result of an influenza virus infection every year [2].

The European Influenza Surveillance Scheme (EISS) is a collaborative network of primary care physicians, epidemiologists and virologists that aims to contribute to a reduction in morbidity and mortality due to influenza in Europe by active clinical and virological surveillance of influenza. Effective influenza surveillance enables an early detection and characterisation of an epidemic as well as the isolation and antigenic characterisation of circulating viruses to assist in the formulation of the following season's vaccine and to provide new vaccine strains $[5,6]$. The participating national reference laboratories have functioned within EISS as the Community Network of Reference Laboratories for Human Influenza in Europe (CNRL) since 2003. They report virus detections and identification data to EISS and work on improving the virological surveillance $[7,8]$.

EISS aims to cover all Member States of the European Union (EU), as required by EU Decision 2119/98/EC on the establishment of dedicated surveillance networks for communicable diseases [9]. During the 2006-2007 winter, EISS covered 26 of the 27 current EU countries (except Bulgaria), as well as Norway, Serbia, Switzerland and Ukraine. A total of 38 national influenza reference laboratories participated in EISS.

The identification of circulating viruses and the recognition of virological changes are major tasks for EISS in order to fulfil its early warning function [7]. There is a particular need to detect and monitor the emergence or re-emergence of viruses with pandemic potential, viruses that 'mismatch' with the vaccine strain, and to monitor the clinical impact of circulating viruses in the community.

During the winter period (week 40 to week 20 of the following year) a Weekly Electronic Bulletin is published each Friday on the EISS website (www.EISS.org), which allows members, public health authorities and the general public to view influenza activity in all participating countries.

This paper presents an analysis and interpretation of influenza surveillance data collected by European countries that were members of EISS during the 2006-2007 winter.

\section{Methods}

\section{Population}

All 30 countries that were members of EISS during the 2006-2007 winter actively monitored influenza activity from about week 40/2006 to about week 20/2007 (Table below). In this paper, England, Northern Ireland, Scotland and Wales are referred to as 
countries as they have their own surveillance systems, and thus we considered EISS to include 33 countries. The characteristics of the sentinel networks are summarised in table 1 of the article supplement. The median weekly population under clinical surveillance by the sentinel networks during the 2006-2007 winter varied from $0.4 \%$ to $100 \%$ of the total population of a country, representing a median number of 30.8 million inhabitants of Europe. In total, about 25,500 general practitioners (GPs), paediatricians and other physicians participated in the sentinel surveillance during the 2006-2007 winter. However, the weekly number of physicians that actually reported was often lower. In general, the age distribution of the population under surveillance was representative for the age distribution of the total population in a country. However, in some countries the population under surveillance was skewed to the lower ages (partly due to a high proportion of paediatricians) and/or higher ages. Further information on the representativeness of the population under surveillance in EISS can be found for most countries in Aguilera et al. [10].

T A B L E

Overview of influenza activity in European countries during the 2006-2007 winter ${ }^{1}$

\begin{tabular}{|c|c|c|c|c|c|c|}
\hline $\begin{array}{l}\text { Country } \\
(\mathrm{N}=33)\end{array}$ & $\begin{array}{l}\text { Week of peak } \\
\text { clinical activity }\end{array}$ & $\begin{array}{l}\text { Most affected } \\
\text { age groups }{ }^{2}\end{array}$ & $\begin{array}{c}\text { Intensity } \\
\text { (peak level) }\end{array}$ & $\begin{array}{l}\text { Week(s) of peak } \\
\text { virus detections }\end{array}$ & $\begin{array}{l}\text { Dominant virus } \\
\text { type/subtype }\end{array}$ & $\begin{array}{c}\text { Geographical } \\
\text { spread (peak level) }\end{array}$ \\
\hline \multicolumn{7}{|c|}{ Influenza-like illness: } \\
\hline Austria & 6 & $0-4$ & Medium & 9 & $\mathrm{~A}(\mathrm{H} 3 \mathrm{~N} 2)$ & Local \\
\hline Belgium & 7 & $0-4,5-14$ & Medium & 6 & $\mathrm{~A}(\mathrm{H} 3 \mathrm{~N} 2)$ & Widespread \\
\hline Cyprus & 2 & n.a. & n.a. & n.a. & n.a. & n.a. \\
\hline Czech Republic & 6 & $0-4,5-14$ & Medium & 6 & $\mathrm{~A}(\mathrm{H} 3)$ & Widespread \\
\hline Denmark & 10 & $0-4$ & High & 9 & $\mathrm{~A}(\mathrm{H} 3 \mathrm{~N} 2)$ & Widespread \\
\hline England & 7 & $15-64,0-4,5-14$ & Medium & 7 & $\mathrm{~A}(\mathrm{H} 3)$ & Regional \\
\hline Estonia & 10 & $5-14,0-4$ & High & 9 & $\mathrm{~A}(\mathrm{H} 3 \mathrm{~N} 2)$ & Widespread \\
\hline Finland & n.a. & n.a. & n.a. & n.a. & $\mathrm{A}$ & n.a. \\
\hline Greece & 3 & n.a. & Medium & 3 & $A(H 3 N 2)$ & Local \\
\hline Hungary & 6 & n.a. & Medium & 7 & $\mathrm{~A}(\mathrm{H} 3)$ & Local \\
\hline Ireland & 7 & $15-64,0-4,5-14$ & Medium & 7 & $\mathrm{~A}(\mathrm{H} 3)$ & Local \\
\hline Italy & 8 & $15-64$ & Medium & 5 & $\mathrm{~A}(\mathrm{H} 3 \mathrm{~N} 2)$ & Widespread \\
\hline Latvia & 9 & $0-4,5-14$ & High & 9 & $\mathrm{~A}(\mathrm{H} 3)$ & Widespread \\
\hline Lithuania & 9 & n.a. & High & 8 & $\mathrm{~A}$ & Regional \\
\hline Luxembourg & 7 & n.a. & High & 6 & $A(H 3 N 2)$ & Widespread \\
\hline Malta & 5 & n.a. & High & n.a. & n.a. & Sporadic \\
\hline Netherlands & 9 & $0-4$ & Medium & 9 & $\mathrm{~A}(\mathrm{H} 3)$ & Widespread \\
\hline Northern Ireland & 5 & n.a. & Medium & 5 & $\mathrm{~A}(\mathrm{H} 3)$ & Sporadic \\
\hline Norway & 8 & $15-64,0-4$ & High & 6 & $\mathrm{~A}(\mathrm{H} 3)$ & Widespread \\
\hline Poland & 9 & $0-4,5-14$ & Medium & 9 & $\mathrm{~A}(\mathrm{H} 1)$ & Sporadic \\
\hline Portugal & 6 & $5-14$ & Medium & 6 & $\mathrm{~A}(\mathrm{H} 3)$ & Widespread \\
\hline Romania & 5 & $0-4$ & Medium & 4 & $\mathrm{~A}(\mathrm{H} 3 \mathrm{~N} 2)$ & Regional \\
\hline Scotland & 2 & n.a. & Medium & 6 & $\mathrm{~A}(\mathrm{H} 3)$ & Regional \\
\hline Serbia & 6 & $0-4,5-14$ & Medium & 6 & $\mathrm{~A}(\mathrm{H} 3)$ & Local \\
\hline Slovakia & 6 & $5-14,0-4$ & Medium & 4 & $\mathrm{~A}(\mathrm{H} 3 \mathrm{~N} 2)$ & Regional \\
\hline Slovenia & 8 & $0-4,5-14$ & Medium & 7 & $A(H 3 N 2)$ & Local \\
\hline Spain & 6 & $5-14,0-4$ & Medium & 5 & $\mathrm{~A}(\mathrm{H} 3 \mathrm{~N} 2)$ & Widespread \\
\hline Sweden & 10 & n.a. & High & $9+10$ & $A(H 3 N 2)$ & Widespread \\
\hline Switzerland & 6 & $5-14,0-4,15-64$ & Medium & 6 & $\mathrm{~A}(\mathrm{H} 3 \mathrm{~N} 2)$ & Widespread \\
\hline Ukraine & n.a. & n.a. & n.a. & 8 & $A(H 1 N 1)+A(H 3 N 2)$ & n.a. \\
\hline Wales & 7 & $15-64$ & Low & 7 & $A$ & Sporadic \\
\hline \multicolumn{7}{|c|}{ Acute respiratory infections: } \\
\hline France & 6 & $0-4,5-14$ & Medium & 5 & $\mathrm{~A}(\mathrm{H} 3 \mathrm{~N} 2)$ & Widespread \\
\hline Germany & 9 & $0-4$ & Medium & 9 & $\mathrm{~A}(\mathrm{H} 3 \mathrm{~N} 2)$ & Widespread \\
\hline
\end{tabular}

1 Sentinel data, except for dominant virus type/subtype for which sentinel and non-sentinel data were taken into account. For definitions of indicators see reference 13 . n.a. = not applicable as no data was available or insufficient data was available. No peak = activity was not above baseline or was flat during the whole winter.
Finland did not report clinical data. Cyprus did not report virological data and Sweden did not report sentinel virological data.

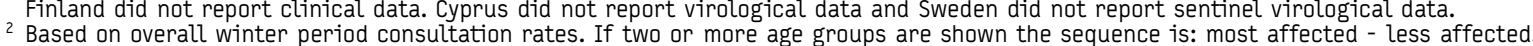

2 Based on overall winter period consultation rates. If two or more age groups are shown the sequence is: most affected - less affected.
3 Estimated where possible taking into account the percentage of influenza virus positive specimens and the absolute number of detections, if the percentage positive specimens was ambiguous only the absolute number of detections was used. 


\section{Clinical surveillance}

In each of the countries except Finland and Ukraine, one or several networks of sentinel physicians reported consultation rates due to influenza-like-illness (ILI) and/or acute respiratory infection (ARI) on a weekly basis. Twenty-seven countries reported ILI consultations per 100,000 population; Malta and Cyprus reported ILI per 100 consultations and France and Germany reported ARI consultations per 100,000 population. In some countries doctors have patient lists, which mean that they have an exact population denominator. For other countries where patients have a free choice of doctors the population denominator has been estimated.

\section{Virological surveillance}

A proportion of the sentinel physicians, in most cases representative for the surveillance network in the country, also collect nose and/or throat swabs for virological surveillance using a swabbing protocol that guarantees representative swabbing during the winter period (table 1 article supplement) [10]. Combining clinical and virological data in the same population allows the evaluation of clinical reports made by the sentinel physicians and provides virological data in a clearly defined population, i.e. the general population that lives in the area served by the participating physician [11]. In addition to specimens obtained from physicians in the sentinel surveillance systems, the laboratories also collect and report results on specimens obtained from other sources (e.g. from hospitals and non-sentinel physicians). These data are called 'non-sentinel' and are collected in order to have a second measure of influenza activity (which contributes to early warning as the entire population is not covered by the sentinel system) and to validate the sentinel virological data [11]. Based on the collection of virological data, the total population under surveillance by EISS, during the winter 2006-2007, was about 497 million inhabitants living in the area covered by EISS [12].

The virological data included results mostly from cell cultures followed by virus type and subtype identification. Rapid diagnostic enzyme-immunological or immunofluorescence tests were also used to identify the virus type only. Many laboratories also use reverse transcription polymerase chain reaction (RT-PCR) routinely for detection, typing and subtyping. Almost 50\% (16/33) of the countries reported antigenic characterisation data and about $30 \%$ $(11 / 33)$ of the countries reported genetic characterisation data of the virus isolates during the 2006-2007 winter.

In addition to the circulation of the seasonal human influenza viruses, EISS laboratories monitored the occurrence of transmission of the highly pathogenic avian influenza virus $A(H 5 N 1)$ to humans in the countries covered by EISS.

\section{Indicators}

During the winter period, the weekly clinical and virological data were collected and analysed by the national centres and then entered into the EISS database the following week via the internet [13]. The clinical consultation rates, the indicators of influenza activity (the intensity of clinical activity and the geographical spread of influenza), as well as the dominant virus type/subtype circulating in the population were established on a weekly basis by the national coordinators based on agreed definitions that were published previously $[8,14]$ (see Box). The dominant type/subtype for the whole winter period shown in the Table above was estimated per country using the algorithm published previously [14].

\section{Spatial analysis}

A spatial analysis of the timing of peak influenza activity across Europe was carried out using regression analysis of plots of the longitude and latitude of the centre of each country against the week of peak influenza activity of each country, as described previously [15].

\section{Results}

\section{Epidemiological data}

The seasonal influenza epidemic started around 1 January 2007 in Europe, with consultation rates for ILI or ARI above levels seen outside the winter period first reported in Scotland (week 52/2006) (graphs 1 and 2 article supplement). Eight countries reported a high intensity of clinical activity, Denmark in weeks 9-12/2007, Estonia in weeks 8-10/2007, Latvia in weeks 9 and 10/2007, Lithuania in weeks 8-10/2007, Luxembourg in weeks 4-7/2007, Malta in weeks 2-7/2007, Norway in weeks 7-10/2007 and Sweden in weeks 9 and 10/2007 (see Table above). Furthermore, Greece reported a local outbreak of influenza activity in week 40/2006 and Sweden reported an exceptional cluster of influenza $A$ in northern Sweden (graph 2 article supplement). Most countries (21/33) reported a medium maximum intensity. Only one country (Wales) reported a low level of intensity throughout the season. Compared to the 2005-2006 winter, the consultation rates for ILI or ARI in the 2006-2007 season were similar in 17 countries that reported these indicators and higher in 12 countries. In particular, in Italy, the consultation rate for ILI in the 2006-2007 winter was much

B o $\mathbf{x}$

Definitions of indicators

\section{Baseline}

Level of clinical influenza activity calculated nationally representing the level of clinical activity in the period that the virus is not epidemic (summer and most of the winter) based on historical data (5-10 influenza seasons).

\section{Intensity}

The intensity of clinical activity compares the weekly clinical morbidity rate with historical data:

- Low $\urcorner-$ no influenza activity or influenza activity at baseline level

- Medium - usual levels of influenza activity

- High - higher than usual levels of influenza activity

- Very high - particularly severe levels of influenza activity (less than once every 10 years

\section{Geographic spread}

The geographical spread is a WHO indicator that has the following levels: - No activity - no evidence of influenza virus activity (clinical activity remains at baseline levels)

- Sporadic - isolated cases of laboratory confirmed influenza infection - Local outbreak - increased influenza activity in local areas (e.g. a city) within a region, or outbreaks in two or more institutions (e.g. schools) within a region, or outbreaks in two or

- Regional activity - influenza activity above baseline levels in one or more regions with a population comprising less than $50 \%$ of the or more regions with a population comprising less

- Widespread - influenza activity above baseline levels in one or more
Widetal regions with a population comprising $50 \%$ or more of the country's population, laboratory confirmed

\section{Dominant virus}

The assessment of the dominant virus for the season is based on: - Sentinel and non-sentinel data (primary assessment sentinel data) - A minimum number of 10 isolates

- If more than $10 \%$ of total $\mathrm{A}$ isolates are $\mathrm{H}$-subtyped the $\mathrm{H}$ subtype is taken into consideration

- If more than $10 \%$ of total $\mathrm{A}$ isolates are $\mathrm{N}$-subtyped the $\mathrm{N}$ subtype is also taken into consideration

- The limits for co-dominant virus types/subtypes are: $45 \%: 55 \%$ 
higher than in the previous season (4,282 in 2006-2007 compared to 243 in 2005-2006) (graph 2 article supplement).

The ILI and ARI consultation rates in Europe reached their peak between week 02/2007 in Scotland and Cyprus and week 10/2007 in Denmark, Estonia and Sweden. ILI and ARI consultation rates started to increase first in the western and south-eastern parts of Europe, then in south-central Europe and finally in the North. Widespread influenza activity was reported across most of Europe by mid-February (week 07/2007). Although influenza activity was still increasing in some countries towards the end of February, in southern and western European countries it started to decline at that time. Clinical influenza activity gradually moved north across Europe and reached its peak around week nine in the Netherlands, Denmark, Germany and Poland, the Baltic states, Norway and Sweden (see Figure 1 below and graph 1 in the article supplement). A similar movement was seen when the timing of peak clinical influenza activity across Europe was analysed. A spatial analysis revealed a significant south-north pattern in the timing of peak

F I G U R E 1

Timing of peak clinical influenza activity across Europe during the 2006-2007 season

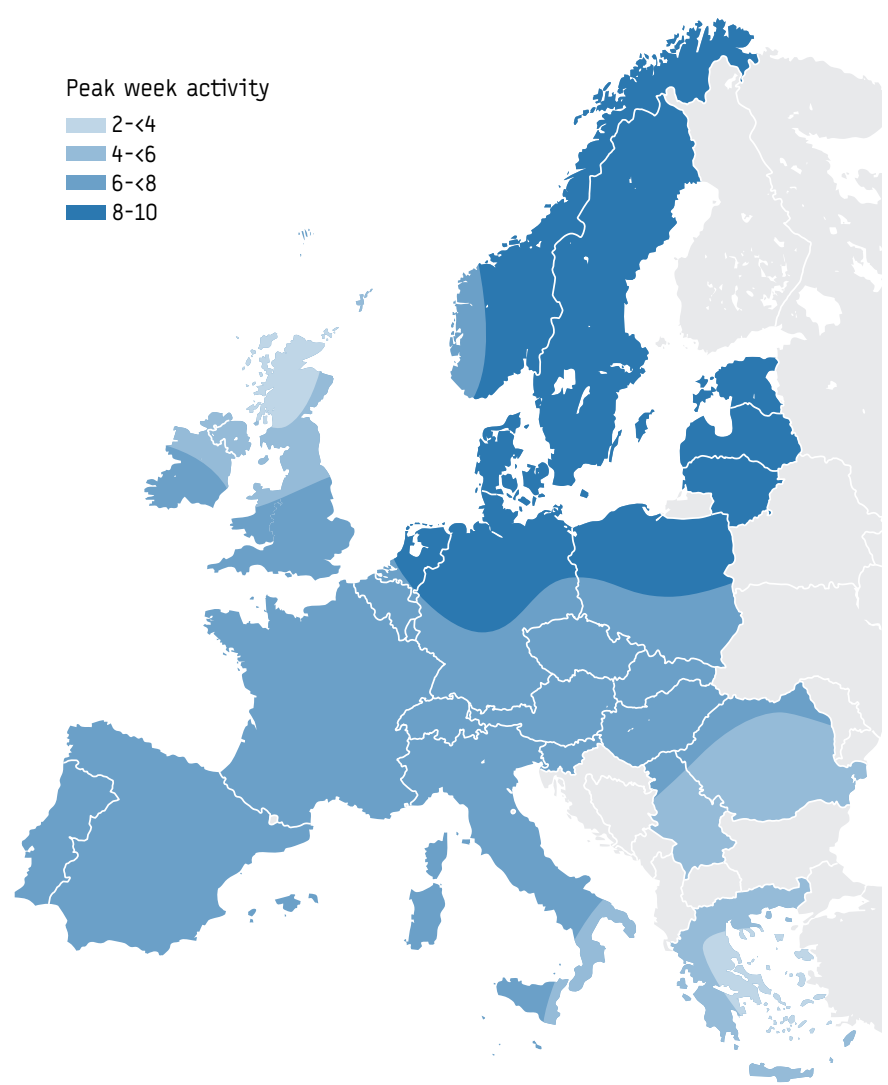

Data source: EISS 2007

Cartography and design: Institute for Hygiene and Public Health University Bonn, Bonn 2007

Note: The isobars on the contour maps represent interpolated time of peak activity distributed spatially at 2 week intervals. Countries included in this spatial analysis were: Austria, Belgium, Czech Republic, Denmark, England, Estonia, France, Germany, Greece, Hungary, Ireland, Italy, Latvia, Lithuania, Luxembourg, Malta, The Netherlands, Norway, Poland, Portugal, Romania, Serbia, Slovakia, Slovenia, Spain, Scotland, Sweden and Switzerland. influenza activity across Europe during the 2006-2007 winter $\left(R^{2}=0.287 ; p<0.05\right.$ for south-north; $R^{2}=0.060$ for west-east) [16]. The timing of peak levels of clinical activity is visualised in Figure 1.

In individual countries, the week of peak ILI/ARI consultation rates coincided roughly with the week of peak sentinel influenza virus detections. In the 29 countries with paired data that could be evaluated the median week of peak ILI/ARI consultation rates was seven (range week $2-10$ ) and the median week of peak virus detections was seven (range week 3 - 10). In 14 (48\%) of the 29 countries, the week of peak consultation rates coincided exactly with the week of peak virus detections. In 24 (83\%) of the 29 countries the week of peak consultation rates either coincided exactly with the week of peak virus detections or the peaks coincided with a difference of one week.

In countries reporting age-specific data $(\mathrm{N}=22)$, the highest consultation rates during the influenza peak were observed among children in the 0-4 years and 5-14 years age groups, although consultation rates in England, Ireland, Italy, Norway and Wales were also high in the 15-64 years age group (see Table for an overview of the influenza activity).

\section{Virological data}

For Europe as a whole, the largest number of influenza virus positive specimens was detected in week 6/2007 ( $N=2,254)$ (Figure 2). A total of 18,278 sentinel and non-sentinel specimens were positive for influenza virus: 17,759 (97\%) were influenza A and 519 (3\%) were influenza B. Of all haemaglutinin-subtyped viruses $(\mathrm{N}=8,934), 8,271$ (93\%) were $\mathrm{H} 3$ and $663(7 \%)$ were $\mathrm{H} 1$. All 4,208 neuraminidase-subtyped $A(H 3)$ viruses were of the $\mathrm{N} 2$ subtype and all 504 neuraminidase-subtyped $A(H 1)$ viruses were of the $\mathrm{N} 1$ subtype. The predominant virus circulating in the individual countries was $A(H 3)$. In Poland $A(H 1)$ was the dominant subtype, in Romania $A(H 3 N 2)$ was co-dominant with $B$ and in Ukraine $\mathrm{A}(\mathrm{H} 1 \mathrm{~N} 1)$ was co-dominant with $\mathrm{A}(\mathrm{H} 3 \mathrm{~N} 2)$ (Table). A relatively high proportion of influenza $B$ viruses were detected in Romania $(45 \%$

\section{F I G U R E}

Number of sentinel and non-sentinel specimens positive for influenza viruses, cumulated for all European countries by week, during the 2006-2007 winter $(\mathrm{N}=18,278$ as of 3 September 2007)

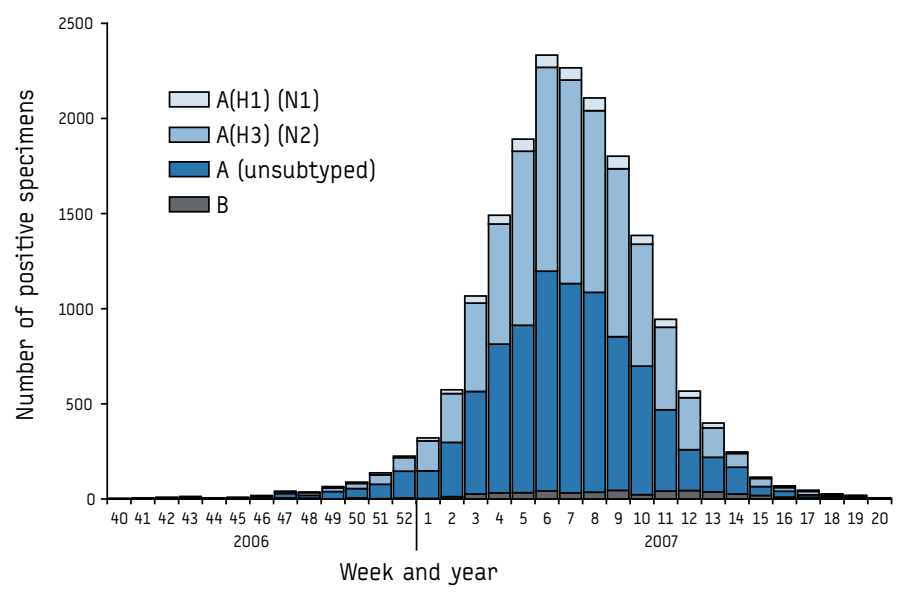


of all influenza viruses) and Ukraine (25\% of all influenza viruses); in all other countries this was $14 \%$ at maximum (in Greece).

Five countries reported laboratory results for detection of the $\mathrm{A}(\mathrm{H} 5 \mathrm{~N} 1)$ virus but none of the 31 specimens from suspected and (possibly) exposed humans tested positive for the $A(H 5 N 1)$ virus. For a detailed breakdown of the virological data for Europe as a whole and by country by week and source (sentinel or non-sentinel) see Figure 3 below, as well as graph 2 and tables 2 and 3 in the article supplement.

Of all 18,278 influenza virus detections, 3,877 have been antigenically and/or genetically characterised: $326(8 \%)$ were A/New Caledonia/20/99 (H1N1)-like, 55 (1\%) were A/California/7/2004 (H3N2)-like, 3,318 (86\%) were A/Wisconsin/67/2005 (H3N2)-like (a drift variant of $A / C a l i f o r n i a / 7 / 2004$ included in the vaccine for the 2006-2007 winter), 148 (4\%) were B/Malaysia/2506/2004-like (B/Victoria/2/87-lineage) and 30 (1\%) were B/Jiangsu/10/2003-like (B/Jiangsu/10/2003 is a B/Shanghai/361/2002-like virus from the $\mathrm{B} /$ Yamagata/16/88-lineage that was included in the vaccine for the 2006-2007 winter).

F I G U R E 3

Breakdown of virus detections cumulated for all European countries by source (sentinel or non-sentinel) and by virus type and subtype, during the 2006-2007 winter

\section{Sentinel virological data $(\mathrm{N}=8,070)$}

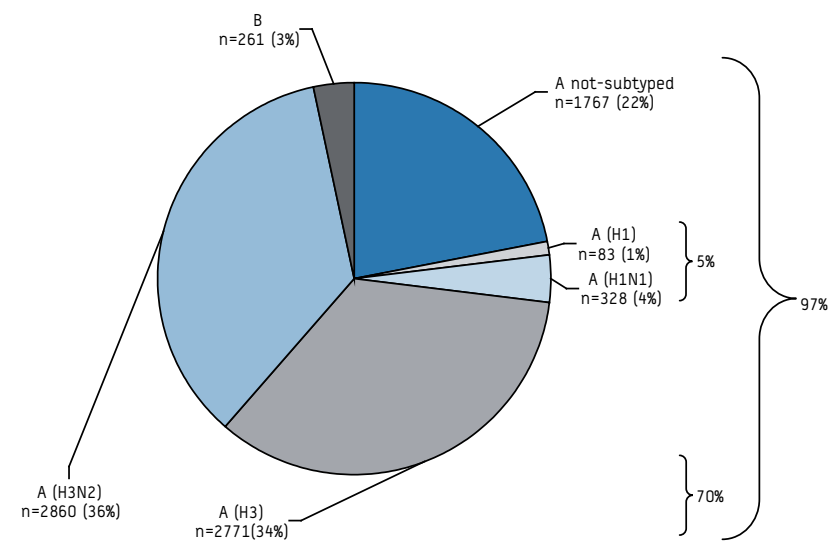

Non-sentinel virological data $(\mathrm{N}=10,208)$

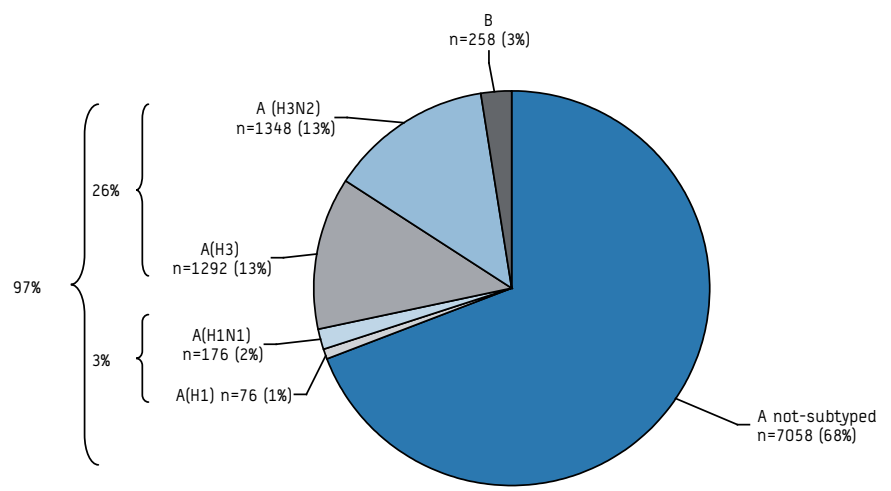

\section{Discussion}

The 2006-2007 influenza season was moderate in Europe in comparison to previous years and was predominantly due to influenza $A$ infections, subtype $\mathrm{H} 3$, with a homogenous spread of viruses across Europe. Influenza activity in Europe started to increase around 1 January 2007 , which is earlier than in the previous winter, when influenza activity in Europe began late in January 2006 . The peak clinical influenza activity by country was for the majority of countries (17/29 countries) similar to the 2005-2006 season, a season dominated by influenza B. For 12 out of 29 countries the peak clinical influenza activity was higher than in the 2005-2006 season. No country had a lower level of influenza activity. The higher peak levels of clinical activity in 2006-2007 compared to 2005-2006 can be attributed to the influenza $A(H 3)$ virus, which usually causes more severe disease symptoms, compared to the influenza $B$ virus and the influenza $A(H 1 N 1)$ virus [4]. The total number of virus detections was $18,278,61 \%$ more than the 11,303 detections during the 2005-2006 season when influenza B was dominant [14].

Compared to other seasons that were dominated by influenza $A(H 3)$ (e.g. the 2004-2005 season), the peak clinical influenza activity for 2006-2007 was similar or lower in the majority of countries (21/25 countries) [15]. The 2006-2007 season lasted from week $02 / 2007$ to week $10 / 2007$, which is relatively short compared to the previous seven seasons, when it lasted from 12 (1999-2000 season) to 19 (2003-2004 season) weeks [16]. Taking into account the relatively low clinical influenza activity and the relatively short duration, the 2006-2007 winter can be considered moderate compared to previous seasons dominated by influenza A.

For Europe as a whole, the 2006-2007 season showed a relatively homogeneous distribution of virus (sub)types across Europe with a dominance of influenza $A(H 3)$ virus. Only in some countries in eastern Europe, there was a relatively high proportion of influenza $B$ virus (Romania) and $A(H 1)$ virus (Poland and Ukraine). However, in previous seasons, including the 2005-2006 winter, it has been shown that when investigated on a country level, virus type and even $\mathrm{H}$-subtype dominance can be heterogeneous across Europe [14]. These observations stress the importance of analysing national or regional virus distribution data.

For the 2006-2007 winter there was a good correlation between clinical and virological data (an overall match of $83 \%,+/-1$ week) compared to the last eight seasons (an overall match of $72 \%,+/-1$ week) [16]. This result once again emphasizes the strength of the surveillance system in that it combines community-based clinical and virological data.

The direction of movement of increased influenza activity is unpredictable. In three of the eight preceding winters there was a south-north movement in the timing of peak influenza activity in countries across Europe [16]. The winter of 2006-2007 tended to fit into this pattern with a northward movement only becoming significant late in the season (Figure 1, graph 1 in the article supplement). Single clusters of influenza outbreak very early in the season, i.e. in Greece (week 51/2006) and Sweden (week 40/2006), did not succeed in country-wide spread of influenza, probably because conditions for further spread were not favourable. In Sweden a hypothesis about the contributions of local temperature and humidity to local epidemics in the north of the country is under investigation (personal communication Urban 
Kumlin, Umeå University. It has been shown that type, subtype and antigenic characteristics of the founder virus, humidity, temperature, UV radiation and air traffic can drive the direction of the movement [17].

Influenza $\mathrm{A}(\mathrm{H} 3)$ viruses that circulated in the 2006-2007 season were antigenically closely related to the A/Wisconsin/67/2005-like vaccine viruses. Similarly, most of the influenza $A(H 1)$ viruses were antigenically closely related to the $2006-2007$ vaccine virus $A /$ New Caledonia/20/99-like vaccine virus. There were no detections of $\mathrm{A}(\mathrm{H} 1 \mathrm{~N} 2)$ in Europe and worldwide observations also suggest that $A(H 1 N 2)$ viruses have become extinct [18]. The majority of circulating influenza $B$ viruses were of the B/Victoria/2/87 lineage and were antigenically and genetically closely related to the 2006-2007 vaccine strain B/Malaysia/2506/2004. To conclude, in the 2006-2007 winter in Europe there was in general a good match between the circulating influenza viruses and the vaccine strains.

In February 2007, The World Health Organization announced the composition of the influenza vaccine for the northern hemisphere to be used for the 2007-2008 influenza season [18]. Based on the available data on the recent influenza viruses provided from all over the world, the WHO modified the recommended composition of the 2007-2008 influenza vaccine compared to the 2006-2007 vaccine. The emergence of a different antigenic variant of $\mathrm{A}(\mathrm{H} 1 \mathrm{~N} 1)$ during the 2006-2007 season prompted the WHO to update the vaccine composition to include the $\mathrm{A}(\mathrm{H} 1 \mathrm{~N} 1) \mathrm{A} / \mathrm{S}$ olomon Islands/3/2006-like $A(H 1 N 1)$ strain. The recommendations for the vaccine reference strains for $A(H 3 N 2)$ and $B$ virus remained the same. The European Agency for the Evaluation of Medicinal Products (EMEA) adopted the recommendations of the WHO [19].

In conclusion, the 2006-2007 influenza epidemic in Europe was characterised by moderate clinical activity and a south-north spread pattern across Europe. The dominant virus strain was influenza $A(H 3)$, and overall there was a good match between the vaccine virus strains and the circulating virus strains.

\section{Contributors}

The members of EISS contributed by weekly submission of influenza surveillance data to EISS during the 2006-2007 winter. JMS Arkema, TJ Meerhoff, A Meijer and WJ Paget carried out weekly analyses of the data and published the Weekly Electronic Bulletins during the 2006-2007 winter. JMS Arkema carried out the overall analysis of the data and prepared the body of the manuscript. A Meijer carried out the analysis for animations of the timeline of increased intensity of influenza activity and national geographic spread of influenza (Graph 1 article supplement). J van der Velden, as chair person of EISS, contributed by supporting the daily operation of EISS during the 2006-2007 winter.

\section{Acknowledgements}

The authors thank F Ansaldi, U Buchholz, D Fleming and staff of the European Centre for Disease Prevention and Control for critically reviewing and commenting the Weekly Electronic Bulletins published during the 2006-2007 winter. EISS would not be able to monitor influenza activity throughout Europe without the commitment and participation of sentinel physicians across Europe. We would like to thank them for making this surveillance scheme possible. In addition we would like to thank all participating institutions during the 2006-2007 winter (for a full list, see article supplement). The authors thank I Wienand from the University of Bonn for carrying out the Kriging analysis of the timing of peak clinical activity across Europe and providing Figure 1.

\section{Article supplement}

The article supplement is available at: http://www.eiss.org/documents/ eurosurveillance_supplement_2006-2007_winter.pdf

The article supplement contains:

i) Lists of persons and institutes participating in EISS during the 2006-2007 winter period,

ii) Characteristics of the influenza surveillance networks in EISS,

iii) Animations of the timing of the change of the clinical intensity and geographic spread indicators by country in Europe,

iv) Graphs of the weekly consultation rates and virus detections by country, and

v) Tables with a detailed breakdown by country of the virological data from sentinel and non-sentinel sources.

References

1. Department of Health, Centres for Disease Control and Prevention, Flu Facts. 2006-2007. Available from: http://www.cdc.gov/flu/keyfacts.htm

2. World Health Organization. Influenza factsheet. 2007. Available from: http:// www.who.int/mediacentre/factsheets/fs211/en/

3. Fleming DM. The contribution of influenza to combined acute respiratory infections, hospital admissions, and deaths in winter. Commun.Dis.Public Health 2000;3(1):32-8.

4. Thompson WW, Shay DK, Weintraub E, Brammer L, Cox N, Anderson LJ, et al. Mortality associated with influenza and respiratory syncytial virus in the United States. JAMA. 2003;289(2):179-86.

5. Aymard M, Valette $M$, Lina B, Thumar B, Members of Groupe Regional d'Observation de la Grippe and European Infuenza Surveillance Scheme. Surveillance and impact of influenza in Europe. Vaccine. 2006;17:S30-S41

6. Fleming DM, van der Velden J, Paget WJ. The evolution of influenza surveillance in Europe and prospects for the next 10 years. Vaccine 2003;21(16):1749-53.

7. Meijer A, Valette M, Manuguerra J, Perez-Brena P, Paget J, Brown C. Implementation of the community network of reference laboratories for human influenza in Europe. J Clin Virol. 2005;34:87-96.

8. Meijer A, Brown C, Hungnes 0 , Schweiger B, Valette M, van der Werf S, et al. Programme of the Community Network of Reference Laboratories for Human Influenza to Improve Influenza Surveillance in Europe. Vaccine. 2006;24(44-46):6717-23.

9. European Commission Directorate-General Health and Consumer Protection Decision 2119/98/EC of the European Parliament and of the Council of 24 September 1998: Setting up a network for epidemiological surveillance and control of communicable disease in the community. Official Journal of the European Commission 1998;8:L268-1-L268/7.

10. Aguilera JF, Paget JW, Manuguerra JC, on behalf of the European Influenza Surveillance Scheme and EuroGROG. Survey of influenza surveillance systems in Europe. Utrecht, the Netherlands: NIVEL, December 2001. Available from: http://www.eiss.org/documents/eiss_inventory_survey_dec_2000.pdf

11. Fleming DM, Chakraverti P, Sadler C, Litton P. Combined clinical and virological surveillance of influenza in winters of 1992 and 1993-4. BMJ. 1995;311:290-1.

12. Statistical Office of the European Communities. 2007.

13. Snacken R, Manuguerra JC, Taylor P. European Influenza Surveillance Scheme on the Internet. Methods Inf Med. 1998;37(3):266-70.

14. Meijer A, Meerhoff TJ, Meuwissen LE, Van Der Velden J, Paget WJ, European Influenza Surveillance Scheme (EISS). Epidemiological and virological assessment of influenza activity in Europe during the winter 2005-2006. Euro Surveill. 2007;12(9):pii=733. Available from: http://www.eurosurveillance. org/ViewArticle.aspx?ArticleId=733

15. Meijer A, Paget WJ, Meerhoff TJ, Brown CS, Meuwissen LE, Van Der Velden J, European Influenza Surveillance Scheme (EISS). Epidemiological and virological assessment of influenza activity in Europe, during the 2004-2005 winter. Euro Surveill. 2006;11(5):pij=623. Available from: http://www.eurosurveillance.org/ ViewArticle.aspx?ArticleId $=623$ 
16. Paget J, Marquet R, Meijer A, van d V. Influenza activity in Europe during eight seasons (1999-2007): an evaluation of the indicators used to measure activity and an assessment of the timing, length and course of peak activity (aspreada) across Europe. BMC Infect Dis. 2007;7(1):141

17. Viboud C, Bjornstad ON, Smith DL, Simonsen L, Miller MA, Grenfell BT. Synchrony, waves, and spatial hierarchies in the spread of influenza. Science. 2006;312:447-51.

18. World Health Organization. Recommended composition of influenza vaccines for use in the 2007-2008 influenza season. Weekly Epidemiological Record 2007;82:69-76

19. World Influenza Centre. Hay A. Influenza between October 2006 to September 2007. Last updated 14 November 2007. Available from: http://www.nimr.mrc. ac.uk/wic/

This article was published on 21 August 2008.

Citation style for this article: Arkema JM, Meijer A, Meerhoff TJ, Van Der Velden J, Paget WJ, European Influenza Surveillance Scheme (EISS). Epidemiological and virological assessment of influenza activity in Europe, during the 2006-2007 winter Euro Surveill. 2008;13(34):pii=18958. Available online: http://www.eurosurveillance. org/ViewArticle.aspx?ArticleId $=18958$ 